\title{
Linfotaping $^{\circledR}$ e o linfedema relacionado ao câncer de mama: uma revisão integrativa
}

\author{
Lymphtaping ${ }^{\circledR}$ and breast cancer-related lymphoedema: an integrative review \\ Lymphtaping $^{\circledR}$ y linfedema relacionado con el cáncer de mama: una revisión integradora
}

Recebido: 20/12/2021 | Revisado: 06/01/2022 | Aceito: 24/01/2022 | Publicado: 25/01/2022

\author{
Mariana Rosado Maia Jales \\ ORCID: https://orcid.org/0000-0002-7193-3129 \\ Universidade do Estado do Rio Grande do Norte, Brasil \\ E-mail: marirosadomj@yahoo.com.br \\ Suyane Bezerra Mota \\ ORCID: https://orcid.org/0000-0001-8034-4978 \\ Universidade do Estado do Rio Grande do Norte, Brasil \\ E-mail: suyanemota@alu.uern.br \\ Micássio Fernandes de Andrade \\ ORCID: https://orcid.org/0000-0003-2836-9080 \\ Universidade do Estado do Rio Grande do Norte, Brasil \\ E-mail: micassiofernandes@gmail.com \\ Ellany Gurgel Cosme do Nascimento \\ ORCID: https://orcid.org/0000-0003-4014-6242 \\ Universidade do Estado do Rio Grande do Norte, Brasil \\ E-mail: ellanygurgel@uern.br \\ Thales Allyrio Araújo de Medeiros Fernandes \\ ORCID: https://orcid.org/0000-0003-4559-8918 \\ Universidade do Estado do Rio Grande do Norte, Brasil \\ E-mail: thalesallyrio@yahoo.com.br
}

\begin{abstract}
Resumo
Introdução: O linfedema relacionado ao câncer de mama (LRCM) é uma condição crônica que pode surgir em decorrência de alterações na estrutura e função linfática provenientes do tratamento desta neoplasia. $\mathrm{O}$ tratamento padrão-ouro desta sequela é a terapia física complexa (TFC); entretanto, pode haver dificuldade de adesão a esta terapêutica por fatores como temperatura, aplicação e estética. Nesse contexto, o Linfotaping ${ }^{\circledR}$ é uma terapêutica alternativa. Objetivo: Avaliar a eficiência do Linfotaping ${ }^{\circledR}$ no tratamento do LRCM, analisando os seus efeitos na qualidade de vida, volume e funcionalidade dos braços afetados. Metodologia: Trata-se de uma revisão da literatura, com busca sistemática, nas bases PubMed, Web of Science, Embase, Scopus, PEDro, SciELO e LILACS. Os termos de busca foram "Breast Cancer Lymphedema, Athletic Tape, Rehabilitation, Recovery of Function, Quality of Life e Clinical Laboratory Techniques". Inicialmente, obtiveram-se 8.363 publicações iniciais. Após a eliminação das duplicatas e aplicação dos critérios de inclusão e exclusão ficaram 17 artigos. Resultados: o Linfotaping ${ }^{\circledR}$ pode promover benefícios na qualidade de vida e no volume e funcionalidade dos braços acometidos pelo LRCM, apesar de haver resultados conflitantes que podem estar relacionados às diferenças metodológicas dos artigos analisados. Conclusão: Alguns estudos mostraram que o Linfotaping ${ }^{\circledR}$ se apresentou como uma alternativa terapêutica interessante para $o$ LRCM. No entanto, faz-se necessária a obtenção de evidências mais sólidas, com a utilização de ensaios clínicos randomizados adicionais, amostragem maior, populações melhor definidas, metodologias mais descritivas e a utilização de outros parâmetros clínicos e laboratoriais para avaliar o efeito da terapia.
\end{abstract}

Palavras-chave: Linfedema relacionado a câncer de mama; Fita atlética; Reabilitação; Recuperação de função fisiológica; Qualidade de vida.

\begin{abstract}
Introduction: The lymphedema related to breast cancer (LRCM) is a chronic condition that can arise due to changes in structure and lymphatic function from the treatment of this neoplasm. The gold standard treatment of this sequel is complex physical therapy (CBT); however, there may be difficulty in adhering to this therapy due to factors such as temperature, application and aesthetics. In this context, Lymphotaping ${ }^{\circledR}$ is an alternative therapy. Objective: To evaluate the efficiency of Linfotaping ${ }^{\circledR}$ in the treatment of CRIM, analyzing its effects on quality of life, volume and functionality of the affected arms. Methodology: This is a literature review, with a systematic search in PubMed, Web of Science, Embase, Scopus, PEDro, SciELO and LILACS. The search terms were "Breast Cancer Lymphedema, Athletic Tape, Rehabilitation, Recovery of Function, Quality of Life and Clinical Laboratory Techniques". Initially, 8,363 publications were obtained. After eliminating the duplicates and applying the inclusion and exclusion criteria, 17 articles remained. Results: Lymphotaping ${ }^{\circledR}$ may promote benefits in quality of life and in the volume and functionality of the arms affected by SCIM, although there are conflicting results that may be related to the methodological differences of the articles analyzed. Conclusion: Some studies showed that Linfotaping $₫$ was an interesting therapeutic
\end{abstract}


alternative for SCIM. However, more solid evidence is needed, with the use of additional randomized clinical trials, larger samples, better defined populations, more descriptive methodologies and the use of other clinical and laboratory parameters to evaluate the effect of therapy.

Keywords: Breast cancer lymphedem; Athletic tape; Rehabilitation; Recovery of function; Quality of life.

\section{Resumen}

Introducción: El linfedema relacionado con el cáncer de mama (LRCM) es una condición crónica que puede surgir debido a los cambios en la estructura y la función linfática del tratamiento de esta neoplasia. El tratamiento de referencia de esta secuela es la fisioterapia compleja (CBT); sin embargo, puede haber dificultades para cumplir con esta terapia debido a factores como la temperatura, la aplicación y la estética. En este contexto, el Lymphotaping® es una terapia alternativa. Objetivo: Evaluar la eficacia del Lymphotaping ${ }^{\circledR}$ en el tratamiento de la CRIM, analizando sus efectos sobre la calidad de vida, el volumen y la funcionalidad de los brazos afectados. Metodología: Se trata de una revisión bibliográfica, con búsqueda sistemática en PubMed, Web of Science, Embase, Scopus, PEDro, SciELO y LILACS. Los términos de búsqueda fueron "Breast Cancer Lymphedema, Athletic Tape, Rehabilitation, Recovery of Function, Quality of Life and Clinical Laboratory Techniques". Inicialmente se obtuvieron 8.363 publicaciones. Tras eliminar los duplicados y aplicar los criterios de inclusión y exclusión, quedaron 17 artículos. Resultados: El Lymphotaping® puede promover beneficios en la calidad de vida y en el volumen y funcionalidad de los brazos afectados por el SCIM, aunque existen resultados contradictorios que pueden estar relacionados con las diferencias metodológicas de los artículos analizados. Conclusión: Algunos estudios mostraron que el Lymphotaping® era una alternativa terapéutica interesante para el SCIM. Sin embargo, se necesitan pruebas más sólidas, con el uso de ensayos clínicos aleatorios adicionales, muestras más grandes, poblaciones mejor definidas, metodologías más descriptivas y el uso de otros parámetros clínicos y de laboratorio para evaluar el efecto de la terapia.

Palabras clave: Linfedema del cáncer de mama; Cinta atlética; Rehabilitación; Recuperación de la función; calidad de vida.

\section{Introdução}

O câncer de mama é a neoplasia mais comum em mulheres em todo o mundo (excluindo-se os de pele não melanoma), e a quantidade de pessoas diagnosticadas com este tipo de tumor continua a crescer. Os avanços científicos relacionados ao tratamento do câncer de mama vêm aumentando a sobrevida desses pacientes, mas pode, em algumas conjunturas, contribuir para o aparecimento de sequelas - como o linfedema (Kasawara et al., 2018; Leung N et al., 2015; Smile TD et al., 2018).

O linfedema relacionado ao câncer de mama (LRCM) é uma morbidade crônica e progressiva, que surge em decorrência de alterações na estrutura e função linfática, caracterizando-se pela concentração de linfa e macromoléculas nos espaços intersticiais, influenciando no sistema imunológico local e geral. O tecido subcutâneo do membro acometido enfrenta, também, um aumento do tecido adiposo patológico, que pode promover o aparecimento de aderências e fibroses no espaço intersticial, e diminuir a flexibilidade desse sistema; e uma infiltração de células inflamatórias e infecções recorrentes, que por sua vez contribuem para a disfunção linfática contínua em um ciclo vicioso (Leung et al., 2015; Azhar et al., 2020; Ezzo et al., 2015; Finnane et al., 2015; Gatt et al., 2017; Invernizzi et al., 2020; Jensen et al., 2015; Li et al., 2020; Ly et al., 2017; Poage et al., 2015; Smoot et al., 2015; Yuan et al., 2019).

O LRCM acomete de 10 a 50\% dos pacientes submetidos ao tratamento do câncer de mama: a incidência varia de acordo com o tipo de abordagem axilar realizada, linfonodos axilares positivos, região de aplicação da radioterapia, complicações no período pós-operatório, obesidade e tempo de sobrevida (Leung et al., 2015; Smile et al., 2018; Ezzo et al., 2015; Li et al., 2016; Singh et al., 2016; Thomaz et al., 2018; Wetzig et al., 2017).

Os principais sinais e sintomas relacionados ao linfedema são as alterações no membro superior, tronco e região peitoral (ou mama residual) ipsilateral à cirurgia da mama: edema; dor; sensação de peso ou aperto; mudança no aspecto da pele e doenças dermatológicas frequentes; e diminuição na amplitude de movimento das articulações desse membro superior. (Ezzo et al., 2015; Li et al., 2016).

O risco de infecções recorrentes na região acometida e as limitações funcionais diárias ocasionadas pelo LRCM favorecem os distúrbios na imagem corporal e a sensação de não recuperação da doença, podendo evoluir para alterações psicológicas como ansiedade e depressão. Consequentemente, há um impacto negativo na qualidade de vida desses sujeitos 
(Leung et al., 2015; Finnane et al., 2015; Gatt et al., 2017; Poage et al., 2015; Smoot et al., 2015; Singh et al., 2016; Thomaz et al., 2018).

A terapêutica para o LRCM enseja uma abordagem interdisciplinar, na qual a fisioterapia tem papel fundamental. O tratamento fisioterapêutico padrão-ouro é a terapia física complexa (TFC), que se divide em duas fases: redução do volume do membro (fase I) - cuidados com a pele, drenagem linfática manual, enfaixamento compressivo multicamadas e exercícios linfomiocinéticos; manutenção do volume do membro (fase II) - cuidados com a pele, auto drenagem linfática, veste compressiva e exercícios linfomiocinéticos. (Ezzo et al., 2015; Finnane et al., 2015; Rezende et al., 2018).

A TFC é eficaz para no tratamento do linfedema, mas há uma dificuldade de adesão aos protocolos propostos, principalmente por questões relacionadas ao enfaixamento compressivo multicamadas (estética e aumento da temperatura local da pele em cerca de $3^{\circ}$ à $5^{\circ} \mathrm{C}$ ) e à frequência de aplicação da técnica. Essa relutância em aderir à TFC pode agravar o quadro e, por isto, se faz necessário avaliar novas alternativas terapêuticas, como o Linfotaping ${ }^{\circledR}$ (Ezzo et al., 2015; Gatt et al., 2017; Poage et al., 2015; Marx \& Figueira, 2017).

Este recurso baseia-se na técnica Kinesio Taping ${ }^{\circledR}(\mathrm{KT})$, que foi desenvolvida pelo terapeuta japonês Kenzo Kase com o objetivo inicial de prevenir ou tratar lesões musculoesqueléticas através do suporte muscular sem restrição dos movimentos. O KT tornou-se amplamente utilizado pelos esportistas japoneses e se difundiu para outros países no final da década de 90, quando passou, então, a ser estudado e utilizado em alterações de outros sistemas - como o linfático (Kasawara et al., 2018, Leung et al., 2015; Gatt et al., 2017; Poage et al., 2015; Smoot et al., 2015; Thomaz et al., 2018; Wallis et al., 2003; Kase, 2003, 2013).

No Linfotaping ${ }^{\circledR}$, usa-se a fita de Kinesio Taping ${ }^{\circledR}$ para promover um alongamento da pele e aumentar o espaço entre a derme e os tecidos subjacentes (convolução), diminuindo a pressão sobre os vasos linfáticos e reduzindo a estase linfática pela diferença de pressão que se forma entre eles e o interstício. Além disso, com os movimentos do corpo, favorece-se a abertura das válvulas dos pequenos vasos linfáticos (Figura 1) (Kasawara et al., 2018; Thomaz et al., 2018; Wallis et al., 2003; Kase, 2003, 2013).

Figura 1. Convolução promovida pelo Linfotaping® (adaptada de Kase K, 2003).
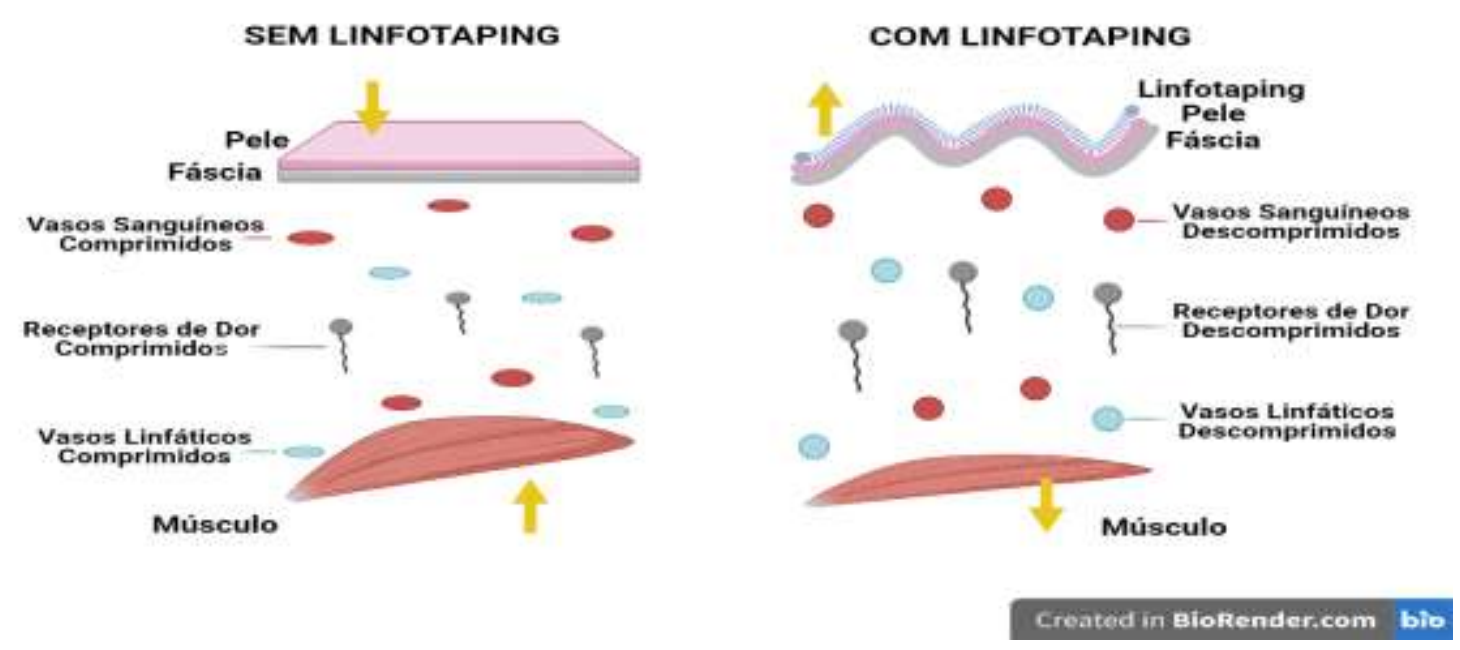

Fonte: BioRender. 
A aplicabilidade do Linfotaping ${ }^{\circledR}$ no tratamento de linfedema relacionado ao câncer de mama vem sendo estudada, mas os resultados ainda são inconclusivos. O objetivo desta revisão é avaliar a eficiência do Linfotaping ${ }^{\circledR}$ no tratamento do LRCM, analisando os seus efeitos na qualidade de vida de mulheres acometidas por esta disfunção, assim como no volume e funcionalidade dos braços afetados por este tipo de linfedema.

\section{Metodologia}

Trata-se de um estudo de revisão de literatura, com busca sistemática, seguindo a pergunta norteadora: Quais os efeitos do Linfotaping ${ }^{\circledR}$ no tratamento do linfedema relacionado ao câncer de mama, em especial na qualidade de vida de mulheres acometidas por esta disfunção, assim como no volume e funcionalidade dos braços afetados por este tipo de linfedema?

Sete bases de dados foram usadas na busca: PubMed, Web of Science, Embase, Scopus, PEDro, SciELO e LILACS. Os descritores foram previamente pesquisados considerando o vocabulário controlado para indexação de artigos do Medical Subject Headings (MeSH), pelo qual foram encontrados: Breast Cancer Lymphedema, Athletic Tape, Rehabilitation, Recovery of Function, e Quality of Life. Eles foram combinados utilizando-se o operador booleano AND da seguinte maneira: Breast Cancer Lymphedema AND Athletic Tape; Breast Cancer Lymphedema AND Rehabilitation; Breast Cancer Lymphedema AND Recovery of Function; Breast Cancer Lymphedema AND Quality of Life.

Foram utilizados como critérios de inclusão pesquisas originais, envolvendo mulheres adultas (maiores de 18 anos), tratadas com Linfotaping ${ }^{\circledR}$ (Kinesio Taping ${ }^{\circledR}$ ) para o linfedema relacionado ao câncer de mama, que analisaram ao menos um dos seguintes aspectos: variação do volume do linfedema, funcionalidade do membro superior comprometido e qualidade de vida. Foram excluídas as revisões sistemáticas, integrativas e meta-análises, editoriais e notas de editor, teses, dissertações e livros. Não foram usadas determinações de língua e tempo.

A pesquisa nos bancos de dados foi realizada entre setembro de 2019 e maio de 2021, e retornou 8.363 artigos. Na sequência, foi usado o provedor "Rayyan Systems Inc.” para identificação e exclusão dos artigos duplicados, e seleção dos artigos, de acordo com os critérios de inclusão e exclusão pré-estabelecidos. Ao final, 17 artigos foram incluídos na revisão publicados entre os anos de 2009 a 2021. As etapas de seleção dos artigos estão descritas na Figura 2.

Posteriormente à seleção dos artigos, foi feito um resumo organizado de seus conteúdos. O processo de extração de dados dos artigos que passaram por triagem foi guiado por uma ficha de análise padrão, elaborada em caráter prévio e empregada na avaliação dos estudos recrutados em todas as estratégias de busca supracitadas. 
Figura 2. Fluxograma da seleção dos artigos para a revisão.
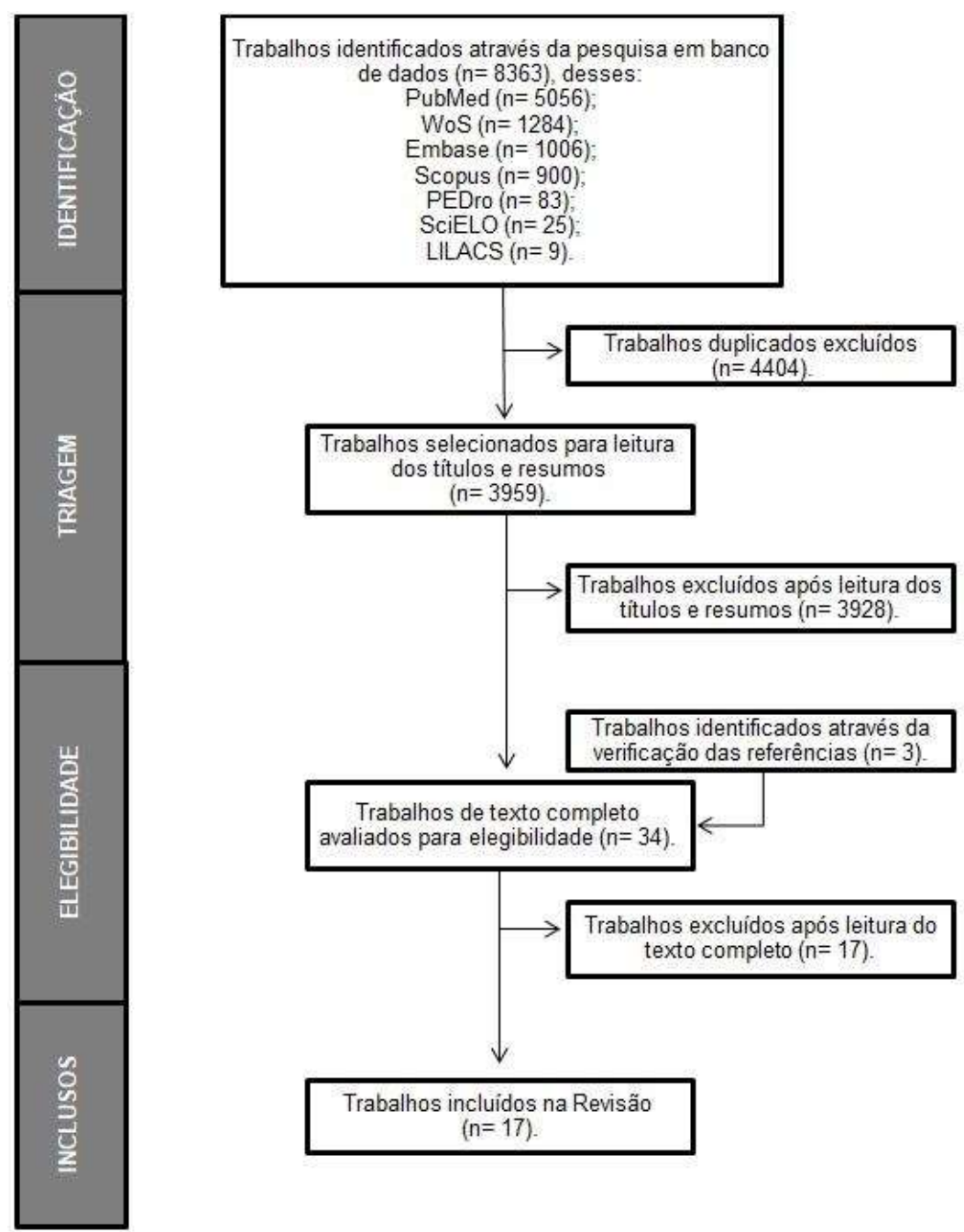

Fonte: Autores.

\section{Resultados}

Foram avaliados os textos completos dos artigos que atenderam aos critérios de inclusão. O esquema PICOT (população, intervenção, comparação, resultado, tipo de estudo) foi utilizado para extrair os dados de interesse dos artigos: características da população (quantas mulheres participaram dos estudos e o grau dos linfedemas), tipo de intervenção (método de aplicação do Linfotaping ${ }^{\circledR}$ e os tipos de tratamentos adicionais), intervenção dos possíveis grupos controles, duração dos tratamentos, os objetivos dos estudos, as variáveis analisadas, os resultados e conclusões. Os dados foram extraídos e documentados sem um software específico e estão descritos nas Tabelas 1, 2 e 3. 
Tabela 1. Dados gerais dos estudos incluídos na revisão.

\begin{tabular}{|c|c|c|c|}
\hline Autores & Tipo de estudo & Objetivos & População \\
\hline Cai H et al., 2021 & $\begin{array}{l}\text { Ensaio clínico } \\
\text { randomizado duplo cego. }\end{array}$ & $\begin{array}{l}\text { Analisar os efeitos clínicos do Kinesio Taping@ combinado com a drenagem } \\
\text { linfática manual em pacientes com linfedema relacionado ao câncer de mama. }\end{array}$ & $\begin{array}{l}70 \text { pacientes com } \\
\text { LRCM. }\end{array}$ \\
\hline Do J et al., 2017 & Estudo de caso. & $\begin{array}{l}\text { Determinar a eficácia do Kinesio Taping® na qualidade de vida, volume e função } \\
\text { do braço de paciente com LRCM, combinado com uma almofada adicional e outras } \\
\text { modalidades terapêuticas padrão para o linfedema. }\end{array}$ & $\begin{array}{l}\text { Uma paciente, com } \\
\text { linfedema estágio } \\
\text { II. }\end{array}$ \\
\hline Ergin G et al., 2019 & $\begin{array}{l}\text { Ensaio clínico } \\
\text { randomizado. }\end{array}$ & Determinar a eficácia do KT nas regiões de anastomoses no manejo do LRCM. & $\begin{array}{l}32 \text { pacientes com } \\
\text { LRCM. }\end{array}$ \\
\hline Malicka I et al., 2014 & $\begin{array}{l}\text { Ensaio clínico } \\
\text { randomizado. }\end{array}$ & Avaliar os efeitos do KT em mulheres com LRCM. & $\begin{array}{l}28 \text { pacientes, com } \\
\text { linfedema grau } 1 .\end{array}$ \\
\hline $\begin{array}{l}\text { de Carvalho Martins } \\
\text { J et al., } 2015\end{array}$ & $\begin{array}{l}\text { Estudo clínico não } \\
\text { randomizado. }\end{array}$ & Avaliar a segurança e a tolerabilidade do KT em pacientes com LRCM. & $\begin{array}{l}24 \text { pacientes com } \\
\text { LRCM. }\end{array}$ \\
\hline $\begin{array}{l}\text { Melgaard D et al., } \\
2016\end{array}$ & $\begin{array}{l}\text { Estudo piloto } \\
\text { randomizado e } \\
\text { controlado. }\end{array}$ & $\begin{array}{l}\text { Identificar se o tratamento para LRCM combinando TFC com KT é tão eficaz } \\
\text { quanto a TFC com compressão de faixas de baixa elasticidade. }\end{array}$ & $\begin{array}{l}10 \text { pacientes com } \\
\text { LRCM estágio II. }\end{array}$ \\
\hline $\begin{array}{l}\text { Pajero Otero V et al., } \\
2019\end{array}$ & $\begin{array}{l}\text { Ensaio Clínico } \\
\text { Randomizado Controlado } \\
\text { Cruzado. }\end{array}$ & $\begin{array}{l}\text { Determinar a Mudança de Volume Relativo (diferença de volume entre os dois } \\
\text { braços) e a Diferença de Volume Relativo (diferença de volume do braço afetado } \\
\text { antes e após tratamento) do linfedema para a intervenção com KT em comparação } \\
\text { com a veste compressiva. E estabelecer a diferença entre as duas intervenções na } \\
\text { amplitude de movimento das articulações dos membros superiores, auto percepção } \\
\text { de conforto e sintomas associados a linfedema (dor, aperto, peso e rigidez). }\end{array}$ & $\begin{array}{l}30 \text { pacientes com } \\
\text { LRCM. }\end{array}$ \\
\hline $\begin{array}{l}\text { Pekyavaş NÖ et al., } \\
2014\end{array}$ & $\begin{array}{l}\text { Ensaio clínico } \\
\text { randomizado. }\end{array}$ & $\begin{array}{l}\text { Investigar os efeitos do KT com a Terapia Descongestiva Complexa em pacientes } \\
\text { com linfedema. }\end{array}$ & $\begin{array}{l}45 \text { pacientes, com } \\
\text { linfedema grau } 2 \text { ou } \\
\quad 3 \text {. }\end{array}$ \\
\hline Pop TB et al., 2014 & $\begin{array}{l}\text { Ensaio clínico } \\
\text { randomizado. }\end{array}$ & Avaliar a influência do Kinesio Taping® no LRCM. & $\begin{array}{l}44 \text { mulheres, com } \\
\text { linfedema estágios I } \\
\text { ou II. }\end{array}$ \\
\hline $\begin{array}{l}\text { Smykla A et al., } \\
2013\end{array}$ & $\begin{array}{l}\text { Ensaio clínico } \\
\text { randomizado. }\end{array}$ & Avaliar a eficácia do KT para o tratamento do LRCM. & $\begin{array}{l}65 \text { mulheres, com } \\
\text { LRCM estágios II } \\
\quad \text { ou III. }\end{array}$ \\
\hline Tantawy, 2019 & Estudo piloto. & Investigar o efeito do KT no LRCM. & $\begin{array}{l}7 \text { mulheres com } \\
\text { LRCM estágios II } \\
\text { ou III. }\end{array}$ \\
\hline $\begin{array}{l}\text { Tantawy \& Kamel, } \\
2016\end{array}$ & $\begin{array}{l}\text { Ensaio clínico } \\
\text { randomizado. }\end{array}$ & $\begin{array}{l}\text { Investigar o efeito do KT na dor, amplitude de movimento e incapacidade do } \\
\text { ombro. }\end{array}$ & $\begin{array}{l}63 \text { mulheres com } \\
\text { dor ou limitação de } \\
\text { movimento após } \\
\text { mastectomia radical } \\
\text { modificada. }\end{array}$ \\
\hline $\begin{array}{l}\text { Tantawy SA et al., } \\
2019\end{array}$ & $\begin{array}{l}\text { Ensaio clínico } \\
\text { randomizado. }\end{array}$ & Comparar os efeitos do KT com a veste compressiva no LRCM. & $\begin{array}{l}59 \text { mulheres com } \\
\text { LRCM. }\end{array}$ \\
\hline Taradaj J et al., 2014 & Estudo de caso. & Discutir o caso de uma mulher com LRCM. & $\begin{array}{l}\text { Uma mulher com } \\
\text { LRCM. }\end{array}$ \\
\hline $\begin{array}{l}\text { Taradaj, J et al., } \\
2015\end{array}$ & $\begin{array}{l}\text { Ensaio clínico } \\
\text { randomizado. }\end{array}$ & $\begin{array}{l}\text { Avaliar o efeito do KT no tamanho do linfedema e na destreza manual do membro } \\
\text { superior em mulheres após o tratamento do câncer de mama. }\end{array}$ & $\begin{array}{l}70 \text { pacientes com } \\
\text { LRCM estágios II } \\
\text { ou III. }\end{array}$ \\
\hline $\begin{array}{l}\text { Torres-Lacomba M } \\
\quad \text { et al., } 2020\end{array}$ & $\begin{array}{l}\text { Ensaio clínico } \\
\text { randomizado, cego. }\end{array}$ & $\begin{array}{l}\text { Comparar a eficácia de quatro tipos diferentes de bandagem com o Kinesio Taping } \\
\text { para o volume do braço com LRCM. }\end{array}$ & $\begin{array}{l}146 \text { com LRCM } \\
\text { estágios I ou II. }\end{array}$ \\
\hline Tsai HJ et al., 2009 & $\begin{array}{l}\text { Ensaio clínico } \\
\text { randomizado, controlado, } \\
\text { cego. }\end{array}$ & $\begin{array}{l}\text { Comparar o efeito da TFC tradicional (com bandagem de baixa elasticidade) } \\
\text { combinada com compressão pneumática e TFC modificada (com KT) combinada } \\
\text { com compressão pneumática. }\end{array}$ & $\begin{array}{l}41 \text { pacientes com } \\
\text { LRCM moderado } \\
\text { ou grave. }\end{array}$ \\
\hline
\end{tabular}

Fonte: Autores. 
Tabela 2. Desenho dos estudos incluídos na revisão.

\begin{tabular}{|c|c|c|c|}
\hline Autores & Intervenção & Comparação /controle & $\begin{array}{l}\text { Tempo de } \\
\text { tratamento }\end{array}$ \\
\hline Cai H et al., 2021 & $\begin{array}{l}\text { Combinação de KT e DLM. } \\
\text { Fita em forma de leque, com origem na região do acrômio e caudas até o } \\
\text { cotovelo Fita em formato de leque, com origem na região interna do cotovelo e } \\
\text { caudas até o centro da palma. Outra fita com origem na região lateral do } \\
\text { cotovelo e inserção nos dedos. Todas com força de tração natural. As fitas foram } \\
\text { aplicadas após os outros tratamentos e removidas no tratamento do dia seguinte }\end{array}$ & $\begin{array}{l}\text { Tratamento convencional: } \\
\text { exercícios para membros } \\
\text { superiores + terapia por pressão } \\
\text { de ondas de ar. }\end{array}$ & $\begin{array}{l}\text { Tratamento } \\
\text { contínuo } 6 \\
\text { dias/semana, por } 4 \\
\text { semanas. }\end{array}$ \\
\hline Do J et al., 2017 & $\begin{array}{l}\text { TFC + almofada + KT. } \\
\text { A fita foi aplicada na mão, braço e tronco, com tração de } 30 \text { a } 40 \% \text {. }\end{array}$ & - & $\begin{array}{l}5 \quad \text { sessões por } \\
\text { semana, durante } 2 \\
\text { semanas. }\end{array}$ \\
\hline Ergin G et al., 2019 & $\begin{array}{l}\text { Grupo } 2(\mathrm{TFC}+\mathrm{KT}) \text {. } \\
\text { O KT foi aplicado nas anastomoses axilo-axilar anterior e posterior e } \\
\text { anastomose axilo-inguinal. A aplicação das anastomoses axilo-axilar iniciou no } \\
\text { lado não afetado e as caudas foram aplicadas até o lado afetado. Para } \\
\text { anastomose axilo-inguinal, a origem foi a região inguinal e caudas até a região } \\
\text { axilar, do lado afetado. Aplicação com nenhuma ou muito pouca tensão (0\% - } \\
15 \%) \text {, e foram deixadas por três dias. }\end{array}$ & Grupo 1 (TFC). & $\begin{array}{l}5 \text { dias por semana, } \\
\text { durante } \\
\text { semanas. }\end{array}$ \\
\hline Malicka I et al., 2014 & $\begin{array}{l}\text { Kinesio Taping (com dois subgrupos de intervenção). } \\
\text { No primeiro subgrupo, a fita foi aplicada em forma de leque no braço e } \\
\text { antebraço e ao longo das anastomoses. No segundo subgrupo, a fita foi aplicada } \\
\text { apenas no braço e antebraço, em forma de leque duplo. A tensão máxima das } \\
\text { caudas foi de } 15 \% \text {. }\end{array}$ & $\begin{array}{l}\text { Nenhum tratamento contra o } \\
\text { linfedema. }\end{array}$ & $\begin{array}{l}1 \text { sessão por } \\
\text { semana, durante } 4 \\
\text { semanas. }\end{array}$ \\
\hline $\begin{array}{l}\text { de Carvalho Martins } \\
\text { J et al., } 2015\end{array}$ & $\begin{array}{l}\text { Kinesio Taping: fita foi aplicada em forma de leque na anastomose axilo-axilar } \\
\text { anterior e posterior, e de proximal para distal no membro superior (oposta à } \\
\text { direção fisiológica do fluxo linfonodal). A bandagem foi aplicada na parte } \\
\text { posterior da mão apenas nas mulheres que apresentavam edema nesta região. A } \\
\text { fita permaneceu por } 4 \text { dias. }\end{array}$ & - & 1 aplicação. \\
\hline $\begin{array}{l}\text { Melgaard D et al., } \\
2016\end{array}$ & Grupo 2: $\mathrm{TFC}+\mathrm{KT}$ & $\begin{array}{l}\text { Grupo 1: TFC com bandagens } \\
\text { de baixa elasticidade. }\end{array}$ & $\begin{array}{l}\text { Grupo 1: } 5 \text { dias / } \\
\text { semana, durante } 4 \\
\text { semanas. } \\
\text { Grupo 2: } 2 \text { dias / } \\
\text { semana, por } 4 \\
\text { semanas. }\end{array}$ \\
\hline $\begin{array}{l}\text { Pajero Otero V et al., } \\
2019\end{array}$ & $\begin{array}{l}\text { KT + Veste Compressiva } \\
\text { Faixas paralelas individuais, começando na axila contralateral, passando pelas } \\
\text { costas do paciente em ondas, e cobrindo o braço até o pulso em um padrão } \\
\text { espiral; sem tensão. Substituído a cada } 7 \text { dias. }\end{array}$ & $\begin{array}{l}\text { Veste Compressiva }+\mathrm{KT} \\
\text { Faixas paralelas individuais, } \\
\text { começando na axila } \\
\text { contralateral, passando pelas } \\
\text { costas do paciente em ondas, e } \\
\text { cobrindo o braço até o pulso em } \\
\text { um padrão espiral; sem tensão. } \\
\text { Substituído a cada } 7 \text { dias. }\end{array}$ & 4 semanas. \\
\hline $\begin{array}{l}\text { Pekyavaş NÖ et al., } \\
2014\end{array}$ & $\begin{array}{l}\text { Grupo 2: KT + TFC. } \\
\text { Grupo 3: KT + TFC (sem a compressão elástica multicamadas). } \\
\text { "Método de Correção Linfática do Kinesio Tape". }\end{array}$ & Grupo 1: TFC. & $\begin{array}{l}1 \text { sessão/dia, } 5 \\
\text { dias por semana, } \\
\text { durante } \\
\text { semanas. }\end{array}$ \\
\hline Pop TB et al., 2014 & $\begin{array}{l}\text { KT + exercícios + educação do paciente. } \\
\text { Método próprio de aplicação do KT. } \\
\text { Aplicação em espiral: fitas em leque, com origem na parte mais distal do } \\
\text { membro e estendeu-se por todo o comprimento do braço. } \\
\text { Aplicação direcional: fitas em forma de leque, caudas em direção aos } \\
\text { linfonodos do lado oposto. } \\
\text { Âncoras sem tensão e caudas com } 10 \% \text { de tensão. }\end{array}$ & $\begin{array}{l}\text { KT + exercícios + educação do } \\
\text { paciente. } \\
\text { Método tradicional: a direção da } \\
\text { aplicação foi da parte proximal } \\
\text { do membro até a mais distante. } \\
\text { As formas de aplicação e o nível } \\
\text { de tensão foram os mesmos do } \\
\text { método próprio. }\end{array}$ & $\begin{array}{l}1 \text { sessão a cada } 7 \\
\text { dias, por } 21 \text { dias. }\end{array}$ \\
\hline
\end{tabular}




\begin{tabular}{|c|c|c|c|}
\hline Smykla A et al., 2013 & $\begin{array}{l}\text { Grupo KT: cuidados com a pele + terapia de compressão pneumática + DLM + } \\
\text { KT. } \\
\text { Âncora da fita na região anterior da mão; caudas nas regiões anterior, medial e } \\
\text { posterior do antebraço e braço, e na parte anterior do peito. Tensão de } 5 \text { a } 15 \% \text {. }\end{array}$ & $\begin{array}{l}\text { Grupo Quasi KT: cuidados com } \\
\text { a pele + terapia de compressão } \\
\text { pneumática + DLM + Quasi KT } \\
\text { (fitas sem efeitos terapêuticos - } \\
\text { emplastros cirúrgicos comuns, } \\
\text { usando a mesma metodologia do } \\
\text { grupo KT). } \\
\text { Grupo MCT: cuidados com a } \\
\text { pele + terapia de compressão } \\
\text { pneumática + DLM + } \\
\text { bandagens de curta extensão } \\
\text { com múltiplas camadas. }\end{array}$ & $\begin{array}{l}3 \text { vezes na } \\
\text { semana, durante } 4 \\
\text { semanas. }\end{array}$ \\
\hline Tantawy, 2019 & $\begin{array}{l}\text { Programa de exercícios domiciliares + KT. } \\
\text { Tórax: âncora na região anterior da axila sadia, fita em leque até a axila afetada. } \\
\text { Tensão das caudas: } 15-20 \% \text {. } \\
\text { Braço: âncora na axila, caudas em leque para as faces anterior, lateral e medial } \\
\text { do braço, com } 15-25 \% \text { de tensão. Âncora na extremidade lateral da clavícula e } \\
\text { estendida pela face posterior do braço. } \\
\text { Antebraço; âncora na região do epicôndilo medial, e caudas na face anterior, } \\
\text { medial e lateral do antebraço. Âncora na região do epicôndilo lateral e caudas } \\
\text { até a face posterior do antebraço. Caudas com 15-25\% de tensão. } \\
\text { Mão: âncora no processo estiloide da ulna e outra âncora no processo estiloide } \\
\text { do rádio. Caudas na superfície dorsal da mão, até as faces laterais e mediais das } \\
\text { articulações interfalangianas proximais, com 15-25\% de tensão. } \\
\text { Todas as âncoras sem tensão. }\end{array}$ & - & $\begin{array}{l}2 \mathrm{x} / \text { semana, por } 3 \\
\text { semanas. }\end{array}$ \\
\hline $\begin{array}{l}\text { Tantawy \& Kamel, } \\
2016\end{array}$ & $\begin{array}{l}\text { Fisioterapia convencional + KT. } \\
\text { Primeira aplicação KT: técnica de inibição, com tensão de } 15 \text { a } 25 \% \text {. } \\
\text { Supraespinhal: âncora logo abaixo da tuberosidade maior do úmero, sem } \\
\text { tensão. Caudas foram aplicadas ao longo do processo espinhoso da escápula e } \\
\text { terminou sem tensão. } \\
\text { Deltóide: forma em Y. Âncora foi aplicada na inserção do deltóide, com a } \\
\text { primeira cauda aplicada ao deltóide anterior e a segunda no deltóide posterior. } \\
\text { As duas caudas terminaram sem tensão. } \\
\text { Redondo Menor: forma em I. Âncora sobre a face anterior da tuberosidade } \\
\text { maior do úmero. Inserção sem tensão no ângulo inferior da escápula. } \\
\text { Segunda aplicação KT: técnica de correção mecânica, com tensão de } 50 \% \text { a } \\
75 \% \text {. Forma em I (cortada ao meio), com origem no processo coracóide, para } \\
\text { o deltóide posterior com pressão descendente. }\end{array}$ & Fisioterapia convencional. & $\begin{array}{l}\text { Os exercícios } \\
\text { foram aplicados } 3 \\
\text { vezes na semana; } \\
\text { o KT foi aplicado } \\
\text { a cada } 3 \text { dias de } \\
\text { intervalo. } \\
\text { Duração de } 2 \\
\text { semanas. }\end{array}$ \\
\hline $\begin{array}{l}\text { Tantawy SA et al., } \\
2019\end{array}$ & $\begin{array}{l}\text { Kinesio Taping (KT). } \\
\text { A aplicação do KT consistia em um formato de leque no tórax: âncora na região } \\
\text { anterior da axila sadia, fita em leque até a axila afetada. Tensão das caudas: 15- } \\
20 \% \text {. } \\
\text { Dois leques no braço: na primeira forma a âncora foi aplicada na axila e caudas } \\
\text { pelas faces anterior, lateral e medial do braço. Na segunda forma, a âncora foi } \\
\text { aplicada na extremidade lateral da clavícula e caudas estendida por pela face } \\
\text { posterior do braço. } \\
\text { Dois leques no antebraço: âncora na região do epicôndilo medial, e caudas na } \\
\text { face anterior, medial e lateral do antebraço. Âncora na região do epicôndilo } \\
\text { lateral e caudas até a face posterior do antebraço. } \\
\text { Mão: âncora no processo estiloide da ulna e outra âncora no processo estiloide } \\
\text { do rádio. Caudas na superfície dorsal da mão, até as faces laterais e mediais das } \\
\text { articulações Inter falangianas proximais. } \\
\text { Âncoras sem tensão e caudas com 15-25\% de tensão. }\end{array}$ & $\begin{array}{l}\text { GP: Veste compressiva }(20-60 \\
\mathrm{mmHg})\end{array}$ & $\begin{array}{l}\text { KT aplicado } 2 \\
\text { vezes na semana; } \\
\text { e Veste } \\
\text { compressiva 15- } \\
18 \text { h de uso por } \\
\text { dia. Duração de } 3 \\
\text { semanas. }\end{array}$ \\
\hline Taradaj J et al., 2014 & $\begin{array}{l}\text { DLM + compressão pneumática + KT. } \\
\text { A âncora foi aplicada na região anterior da mão sem tensão. As caudas, nos } \\
\text { aspectos anterior, medial e posterior do antebraço e braço com 5-15\% de tensão } \\
\text { e, em seguida, na parte anterior do tórax. }\end{array}$ & - & $\begin{array}{l}3 \text { semanas: } 12 \\
\text { DLM; } 12 \\
\text { Compressões } \\
\text { pneumáticas; três } \\
\text { aplicações de KT. }\end{array}$ \\
\hline Taradaj, J et al., 2015 & $\begin{array}{l}\text { Grupo A: KT + DLM + DLP. } \\
\text { As fitas de KT foram aplicadas de sem tensão até } 15 \% \text {, de acordo com a } \\
\text { metodologia de Kenzo Kase: duas aplicações da fossa cubital aos dedos; duas } \\
\text { aplicações da axila ao longo do braço até a fossa cubital; uma aplicação da } \\
\text { região axilar no lado não operado através do tórax para o axilar no lado } \\
\text { comprometido; da região inguinal do lado comprometido ao longo do tronco } \\
\text { até a axila do mesmo lado. }\end{array}$ & $\begin{array}{l}\text { Grupo B: Quasi KT + DLM + } \\
\text { DLP. } \\
\text { Grupo C: DLM + DLP + } \\
\text { enfaixamento compressivo } \\
\text { multicamadas. }\end{array}$ & $\begin{array}{lr}1 \text { sessão ao dia, } 3 \\
\text { vezes na semana, } \\
\text { durante } \quad 4 \\
\text { semanas. KT } \\
\text { aplicado toda } \\
\text { segunda-feira. }\end{array}$ \\
\hline
\end{tabular}


Research, Society and Development, v. 11, n. 2, e28411224973, 2022

(CC BY 4.0) | ISSN 2525-3409 | DOI: http://dx.doi.org/10.33448/rsd-v11i2.24973

\begin{tabular}{|c|c|c|c|}
\hline $\begin{array}{l}\text { Torres-Lacomba M } \\
\text { et al., } 2020\end{array}$ & $\begin{array}{l}\text { Grupo KT: trocado a cada sessão } \\
\text { Leque em forma de "I", com duas caudas: âncora sem tensão e caudas de } 15 \% \\
-20 \% \text { de tensão. } \\
\text { Ombro: } 1^{\mathrm{a}} \text { âncora na região axilar, caudas para as faces anterior, lateral e } \\
\text { medial; } 2^{\mathrm{a}} \text { âncora na extremidade lateral da clavícula e caudas para a face } \\
\text { posterior do braço. } \\
\text { Antebraço e mão: } 1^{\text {a }} \text { âncora no epicôndilo medial e caudas para as faces } \\
\text { anterior, medial e lateral do antebraço; } 2^{\mathrm{a}} \text { âncora no epicôndilo lateral, e caudas } \\
\text { para a face posterior do antebraço, superfície dorsal da mão e para a face medial } \\
\text { e lateral das articulações Inter falangianas proximais. } \\
+ \text { + DLM; compresãa pneumática ( } 40 \mathrm{~mm} \mathrm{Hg} \text { ) e estratégia educacional } \\
\text { terapêutica (instruções sobre anatomia do sistema linfático e fisiopatologia, } \\
\text { prevenção e identificação de fatores de risco de linfedema); exercícios ativos. }\end{array}$ & 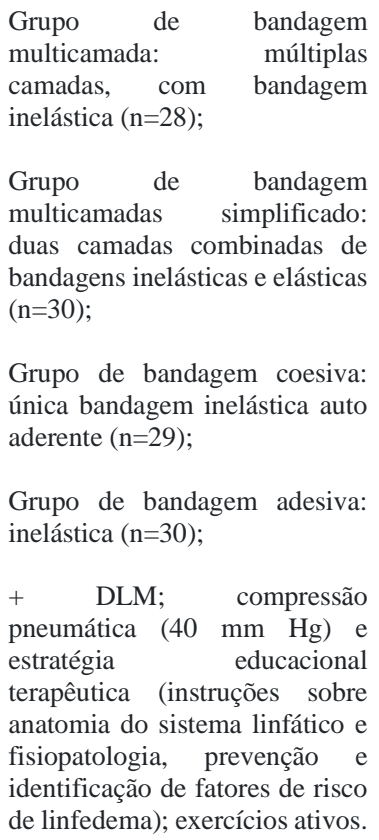 & $\begin{array}{lr}3 \text { semanas } & (2 \\
\text { semanas com } 5 \\
\text { dias consecutivos } \\
\text { de tratamento, } \\
\text { seguido de } 1 \\
\text { semana com } 3 \\
\text { dias alternados de } \\
\text { tratamento). }\end{array}$ \\
\hline Tsai HJ et al., 2009 & $\begin{array}{l}\text { Cuidados com a pele }+ \text { DLM + terapia de compressão pneumática }+ \text { KT }+ \\
\text { exercícios fisioterapêutico. }\end{array}$ & $\begin{array}{l}\text { Cuidados com a pele }+ \text { DLM + } \\
\text { terapia de compressão } \\
\text { pneumática }+ \text { bandagem de } \\
\text { baixa elasticidade }+ \text { exercícios } \\
\text { fisioterapêutico. }\end{array}$ & $\begin{array}{lr}4 \quad \text { semanas } & \text { de } \\
\text { controle } & \text { (sem } \\
\text { tratamento), } & 4 \\
\text { semanas } & \text { de } \\
\text { tratamento } & (5 \\
\text { sessões } & \\
\text { semanais), } & 3 \\
\text { meses } & \text { de } \\
\text { acompanhamento. }\end{array}$ \\
\hline
\end{tabular}

Fonte: Autores. 
Tabela 3. Resultados e Conclusões dos estudos incluídos na revisão.

\begin{tabular}{|c|c|c|}
\hline Autores & Variáveis analisadas & Resultados e Conclusões \\
\hline Cai H et al., 2021 & $\begin{array}{l}\text { Grau do linfedema do braço, } \\
\text { fluxo linfático função do } \\
\text { membro superior e qualidade } \\
\text { de vida. }\end{array}$ & $\begin{array}{l}\text { Houve melhora significativa em todas as variáveis analisadas nos dois grupos, porém no grupo } \\
\text { intervenção a diferença foi significativamente maior. } \\
\text { Os resultados sugerem efeito positivo do tratamento combinado de KT e DLM, digno de aplicação } \\
\text { clínica. }\end{array}$ \\
\hline Do J et al., 2017 & $\begin{array}{l}\text { Qualidade de vida, volume e } \\
\text { função do braço. }\end{array}$ & $\begin{array}{l}\text { Melhorou a qualidade de vida, a capacidade funcional, e reduziu } 79,5 \% \text { no volume do edema. } \\
\text { O uso da almofada e do KT demonstra um resultado positivo no tratamento do LRCM e, portanto, } \\
\text { são sugeridos mais estudos sobre esse método com um tamanho de amostra maior. }\end{array}$ \\
\hline Ergin G et al., 2019 & Volume do braço. & $\begin{array}{l}\text { Os dois grupos apresentaram redução significativa do volume do linfedema após o tratamento, mas } \\
\text { não houve diferença significativa entre eles. } \\
\text { Não há efeito positivo do KT aplicado a regiões de anastomoses na redução do volume dos braços } \\
\text { com LRCM. }\end{array}$ \\
\hline Malicka I et al., 2014 & Volume do braço. & $\begin{array}{l}\text { Redução significativa na extensão do linfedema }(\mathrm{p}=0,0009) \text { no grupo KT, entre as avaliações basal } \\
\text { e pós-tratamento. Nenhuma redução no grupo controle }(\mathrm{p}=0,36) \text {. } \\
\text { KT é um método eficaz de tratamento precoce do edema, e pode ser uma nova opção terapêutica } \\
\text { segura em pacientes contraindicados para o uso de outros métodos. }\end{array}$ \\
\hline $\begin{array}{l}\text { de Carvalho Martins J } \\
\text { et al., } 2015\end{array}$ & $\begin{array}{l}\text { Alterações dermatológicas, } \\
\text { tolerância à técnica, volume e } \\
\text { função dos braços. }\end{array}$ & $\begin{array}{l}\text { 4,2\% das pacientes apresentaram descamação da pele e vermelhidão. A maioria das pacientes não } \\
\text { relatou mudança na vida social, se sentiu mais segura nas atividades diárias e ficou muito satisfeita } \\
\text { com o tratamento. Houve melhora da funcionalidade do membro superior após a intervenção (p } \\
<0,001) \text {. Não foi encontrada diferença no volume dos membros após a intervenção }(\mathrm{p}=0,639) \text {. } \\
\text { A fita de Kinesio Tex Gold aplicada pela técnica KT mostrou-se segura e tolerável em pacientes com } \\
\text { linfedema, com funcionalidade aprimorada e sem alteração do volume do membro afetado. }\end{array}$ \\
\hline $\begin{array}{l}\text { Melgaard D et al., } \\
2016\end{array}$ & $\begin{array}{l}\text { Circunferência do linfedema; } \\
\text { qualidade de vida dos sujeitos } \\
\text { durante o tratamento; custos } \\
\text { dos tratamentos; e ambiente } \\
\text { de trabalho dos fisioterapeutas } \\
\text { durante o tratamento. }\end{array}$ & $\begin{array}{l}\text { Dos } 7 \text { pontos em que foi realizada a circunferência do linfedema, } 4 \text { pontos tiveram melhores } \\
\text { resultados no grupo controle, e } 3 \text { pontos no grupo intervenção; a qualidade de vida é maior, e os } \\
\text { custos e o ambiente de trabalho são melhores no grupo intervenção; porém não é possível documentar } \\
\text { qualquer diferença significativa para as variáveis analisadas. } \\
\text { A TFC com KT pode ser uma alternativa à TFC com bandagem. }\end{array}$ \\
\hline $\begin{array}{l}\text { Pajero Otero V et al., } \\
2019\end{array}$ & $\begin{array}{l}\text { Volume do linfedema; } \\
\text { amplitude de movimento das } \\
\text { articulações dos membros. }\end{array}$ & $\begin{array}{l}\text { A diferença de volume relativo do membro superior mostra uma diferença significativa entre as } \\
\text { intervençes. } \\
\text { Uma mudança de volume relativo mostra que a diferença obtida entre as intervenções foi } \\
\text { significativa. A mudança de volume relativo observada ao longo dos dois períodos de intervenção do } \\
\text { ensaio cruzado foi semelhante em ambos os subgrupos KT e em ambos os subgrupos de vestimentas } \\
\text { de compressão, independentemente da ordem e período. } \\
\text { Não houve mudança significativa durante o período de washout. } \\
\text { Em termos de conforto, o KT foi estatisticamente mais confortável do que a roupa de compressão; } \\
\text { assim como na melhora da amplitude de movimento de flexão e abdução do ombro, flexão do } \\
\text { cotovelo e flexão e extensão do punho; e na percepção de aperto e rigidez. } \\
\text { KT pode ser uma terapia eficaz e confortável para o tratamento do LRCM durante a fase de } \\
\text { manutenção da terapia descongestiva complexa. Deve-se ter cuidado ao retirar as fitas e evitar o uso } \\
\text { prolongado para evitar lesões dermatológicas. São necessárias mais pesquisas com descrição } \\
\text { detalhada dos protocolos para aplicação de KT, e também comparando esta técnica com outros } \\
\text { métodos de aplicação e outras terapias para LRCM. Além disso, a eficácia do KT em outros estágios } \\
\text { do linfedema deve ser explorada para identificar em quais promove mais benefícios. }\end{array}$ \\
\hline $\begin{array}{l}\text { Pekyavaş NÖ et al., } \\
2014\end{array}$ & $\begin{array}{l}\text { Volume dos braços e } \\
\text { qualidade de vida (sintomas } \\
\text { clínicos). }\end{array}$ & $\begin{array}{l}\text { Os sintomas diminuíram nos três grupos }(\mathrm{p}<0,05) \text {. TFC foi efetiva para o volume do braço apenas } \\
\text { durante o tratamento }(\mathrm{p}<0,05) \text {. KT }+ \text { TFC reduziu o volume até } 10 \text { dias após o período de tratamento } \\
(\mathrm{p}<0,05) \text { e para o período controle }(\mathrm{p}<0,05) \text {. Somente a aplicação do KT também apresentou redução } \\
\text { significativa no volume }(\mathrm{p}<0,05) \text {. } \\
\text { A aplicação de } \mathrm{KT}+\mathrm{TFC} \text { pode ter um efeito melhor na diminuição do linfedema, o que pode } \\
\text { estimular a redução do volume para efeitos em longo prazo. }\end{array}$ \\
\hline Pop TB et al., 2014 & $\begin{array}{l}\text { Avaliação subjetiva dos } \\
\text { efeitos terapêuticos; volume } \\
\text { do braço; força de preensão; } \\
\text { amplitude de movimento do } \\
\text { ombro. }\end{array}$ & $\begin{array}{l}\text { Redução de } 55 \% \text { no volume do linfedema, com a técnica espiral produzindo melhores resultados. O } \\
\text { resultado foi significativo em relação ao subconjunto de fitas convencionais (55\% vs. } 27 \% \text { - p } \\
<0,001) \text {. } \\
\text { Na redução do linfedema, foi relatada maior efetividade do método de aplicação próprio em } \\
\text { comparação ao método tradicional. O KT exerceu influência na melhora da mobilidade das } \\
\text { articulações dos membros superiores e na força de preensão. }\end{array}$ \\
\hline
\end{tabular}




\begin{tabular}{|c|c|c|}
\hline Smykla A et al., 2013 & Volume do Braço. & $\begin{array}{l}\text { Comparando os grupos que usaram fitas KT e fita Quasi KT, não houve diferenças significativas (P> } \\
0,05 \text { ). A redução do volume do grupo bandagens multicamadas foi muito melhor do que nos } \\
\text { resultados observados nos grupos de Taping. O KT parece ser ineficaz no LRCM. } \\
\text { Os resultados sugerem que a fita KT não poderia substituir a bandagem compressiva e, neste } \\
\text { momento, não deve ser uma opção alternativa para a paciente com LRCM. }\end{array}$ \\
\hline Tantawy, 2019 & $\begin{array}{l}\text { Soma das circunferências dos } \\
\text { braços. }\end{array}$ & $\begin{array}{l}\text { Os resultados do estudo mostraram significância clínica e estatística do KT no linfedema quando } \\
\text { comparado à pré-aplicação. } \\
\text { O KT é uma abordagem terapêutica avançada no tratamento de indivíduos com LRCM que apresenta } \\
\text { problemas com outras terapias, sendo um procedimento alternativo para outras técnicas. }\end{array}$ \\
\hline $\begin{array}{l}\text { Tantawy \& Kamel, } \\
2016\end{array}$ & $\begin{array}{l}\text { Dor, amplitude de movimento } \\
\text { e função do ombro. }\end{array}$ & $\begin{array}{l}\text { O grupo experimental mostrou diferenças significativas em todas as medidas de resultados, tanto } \\
\text { dentro como entre os grupos. O grupo controle mostrou apenas uma diferença significativa dentro do } \\
\text { grupo na flexão do ombro. } \\
\text { KT pode ser sugerido e recomendado para pacientes pós-mastectomia, principalmente para dor no } \\
\text { ombro, amplitude de movimento e incapacidade. }\end{array}$ \\
\hline $\begin{array}{l}\text { Tantawy SA et al., } \\
2019\end{array}$ & $\begin{array}{l}\text { Volume dos braços; dor e } \\
\text { função do ombro; força de } \\
\text { preensão manual; e qualidade } \\
\text { de vida. }\end{array}$ & $\begin{array}{l}\text { A soma das circunferências dos membros, SPADI, força de preensão manual e qualidade de vida } \\
\text { melhorou significativamente após o tratamento no grupo KT }(\mathrm{P}<0,05) \text {. Enquanto o GP não } \\
\text { apresentou melhora significativa no SPADI, a força de preensão manual, a força física, a função, a } \\
\text { dor e a fadiga pontuaram } p>0,05 \text {, enquanto a soma das circunferências dos membros diminuiu } \\
\text { significativamente }(\mathrm{P}<0,05) \text {. Diferenças significativas foram observadas entre os grupos KT e GP } \\
\text { no final da intervenção }(\mathrm{P}<0,05) \text {. } \\
\text { O KT apresentou alterações significativas na circunferência do membro, SPADI, força de preensão } \\
\text { manual e qualidade de vida geral que o GP no tratamento de indivíduos diagnosticados com } \\
\text { linfedema após mastectomia. }\end{array}$ \\
\hline Taradaj J et al., 2014 & Volume do braço. & $\begin{array}{l}\text { Redução do linfedema }(627 \mathrm{~cm} 3) \text {. } \\
\text { Observou-se que o KT teve um efeito significativo na redução do linfedema e acelerou os efeitos de } \\
\text { cicatrização em comparação com os métodos padrão. }\end{array}$ \\
\hline Taradaj, J et al., 2015 & $\begin{array}{l}\text { Volume do braço, força de } \\
\text { preensão e amplitude de } \\
\text { movimento. }\end{array}$ & $\begin{array}{l}\text { O KT pode ser uma boa opção para pacientes que são resistentes ou têm contra indicações à PC. } \\
\text { Houve redução de } 22,45 \% \text { no volume de membros }(\mathrm{p}=0,000118) \text { e redução de } 24,13 \% \text { no edema (p } \\
=0,00041) \text {. Redução não significativa em relação a outros grupos. } \\
\text { KT não é um método eficaz para reduzir o LRCM em estágios II e III. Ele melhora a amplitude de } \\
\text { movimento nas articulações do membro superior e a força muscular nestes pacientes, mas não mais } \\
\text { do que outros métodos padrão e reconhecidos de terapia linfática descongestiva. Neste momento, o } \\
\text { KT não pode substituir o enfaixamento múltiplas camadas tradicional e padrão no tratamento do } \\
\text { LRCM. }\end{array}$ \\
\hline $\begin{array}{l}\text { Torres-Lacomba M et } \\
\text { al., } 2020\end{array}$ & $\begin{array}{l}\text { Volume e sintomas clínicos } \\
\text { do LRCM (peso e aperto), e } \\
\text { conforto e efeitos adversos } \\
\text { das bandagens e KT. }\end{array}$ & $\begin{array}{l}\text { Foram encontradas diferenças significativas em todos os grupos em relação ao valor absoluto e } \\
\text { redução percentual do volume em excesso }(\mathrm{P}<0,001) \text {, exceto para grupo de bandagem coesiva versus } \\
\text { grupo de bandagem multicamadas simplificado }(\mathrm{P}=0,456) \text {; grupo de bandagem coesiva versus grupo } \\
\text { de bandagem multicamada }(\mathrm{P}=0,792) \text {; grupo de bandagem adesiva versus grupo de bandagem } \\
\text { multicamada }(\mathrm{P}=0,232) \text {; e grupo de bandagem adesiva versus grupo KT }(\mathrm{P}=0,071) \text {. } \\
\text { Em relação aos sintomas de peso e aperto, eles diminuíram significativamente após o tratamento ( } \mathrm{P} \\
=0,031 \text { e } \mathrm{P}=0,026 \text {, respectivamente); no entanto, não houve diferenças significativas entre os } \\
\text { grupos. Quanto ao conforto percebido, o KT foi o mais confortável e a bandagem multicamada a mais } \\
\text { desconfortável (5,3 pontos menos confortável em uma escala numérica de } 11 \text { pontos, } \mathrm{P}<0,001) \text {. } \\
\text { Acerca dos efeitos adversos, dois participantes retiraram as bandagens por desconforto inespecífico } \\
\text { (um no grupo multicamadas e um no grupo coesivo) e um participante apresentou irritação cutânea } \\
\text { (grupo KT). } \\
\text { A bandagem múltiplas camadas simplificada parece mais eficaz e mais confortável do que a } \\
\text { bandagem de múltiplas camadas tradicional, e a bandagem coesa parece tão eficaz quanto a } \\
\text { bandagem de múltiplas camadas simplificada e tradicional, o que pode indicar que o uso de } \\
\text { bandagens de compressão de múltiplas camadas tradicionais na fase de tratamento intensivo do } \\
\text { LRCM em estágios I e II devem ser reconsiderados, com foco no aumento da função do paciente e } \\
\text { da mobilidade geral. }\end{array}$ \\
\hline Tsai HJ et al., 2009 & $\begin{array}{l}\text { Tamanho do braço; } \\
\text { composição da água do braço; } \\
\text { sintomas relacionados ao } \\
\text { linfedema (rigidez, peso, dor, } \\
\text { rigidez, irritabilidade, } \\
\text { desconforto, calor, plenitude, } \\
\text { formigamento, fraqueza e } \\
\text { dormência); qualidade de } \\
\text { vida; resposta dos sujeitos à } \\
\text { bandagem de baixa } \\
\text { elasticidade ou KT (adesão ao } \\
\text { tratamento). }\end{array}$ & $\begin{array}{l}\text { Não houve diferença significativa entre os grupos em todas as variáveis }(\mathrm{P}>0,05) \text { durante todo o } \\
\text { período de estudo. } \\
\mathrm{O} \text { tamanho do braço e a composição da água foram reduzidos significativamente tanto no grupo da } \\
\text { bandagem de baixa elasticidade quanto no grupo do KT. A aceitação do KT foi melhor do que a } \\
\text { bandagem de baixa elasticidade, e os benefícios incluíram maior tempo de uso, menos dificuldade de } \\
\text { uso e maior conforto e conveniência }(\mathrm{P}<0,05) \text {. } \\
\text { Ao comparar o uso da bandagem de baixa elasticidade com o KT em pacientes com LRCM, que } \\
\text { receberam TFC combinada com compressão pneumática, sugere-se que o KT pode substituir a } \\
\text { bandagem para pacientes que tiveram baixa adesão ao uso de bandagem após } 1 \text { mês intervenção. } \\
\text { Além disso, os dois protocolos de tratamento estudados são ineficientes e custam tempo na aplicação. } \\
\text { Um protocolo de tratamento mais eficiente é necessário para a prática clínica. }\end{array}$ \\
\hline
\end{tabular}


Entre os dezessete artigos avaliados, um foi realizado no Brasil, de autoria de Carvalho Martins (2015); cinco deles na Polônia (Malicka et al., 2014; Pop et al., 2014; Smykla et al., 2013; Taradaj et al., 2014; Taradaj et al., 2015), três no Egito (Tantawy, 2019; Tantawy \& Kamel, 2016; Tantawy et al., 2019), dois na República da China (Cai H et al., 2021; Tsai HJ et al., 2009), dois na Turquia (Ergin et al., 2019; Pekyavaş et al., 2014), dois na Espanha (Pajero Otero V et al., 2019; Torres-Lacomba M et al., 2020), um na Dinamarca (Melgaard et al., 2016) e um na República da Coréia (Do J et al., 2017). Há, portanto, uma carência de pesquisas com essa temática que enfoquem a população sul-americana.

Os estudos avaliaram ao todo 732 indivíduos.

O volume do membro acometido pelo LRCM foi a variável predominante, sendo mensurada em quinze estudos: dez deles mostraram resultados positivos (Cai et al., 2021; Do et al., 2017; Malicka et al., 2014; Pajero Otero et al., 2019; Pekyavaş et al., 2014; Pop et al., 2014; Tantawy, 2019; Tantawy et al., 2019; Taradaj et al., 2014; Torres-Lacomba et al., 2020) e cinco não apresentaram significância para o uso do Linfotaping ${ }^{\circledR}$ na redução do volume (Ergin et al., 2019; de Carvalho Martins et al., 2015; Smykla et al., 2013; Taradaj et al., 2015; Tsai et al., 2009).

A função do braço foi analisada em dez pesquisas: oito delas apresentaram resultados positivos (Cai et al., 2021; Do et al., 2017; de Carvalho Martins et al., 2015; Pajero Otero et al., 2019; Pop et al., 2014; Tantawy, 2019; Tantawy \& Kamel, 2016; Tantawy et al., 2019) e dois não encontraram mais benefício do Linfotaping ${ }^{\circledR}$ do que as outras terapias empregadas (Taradaj et al., 2015; Tsai et al., 2009).

A qualidade de vida dos sujeitos foi investigada em nove artigos: seis obtiveram resultados positivos (Cai et al., 2021; Do et al., 2017; Melgaard et al., 2016; Pekyavaş et al., 2014; Tantawy, 2019; Tantawy et al., 2019); dois deles indicam resultados benéficos para fatores que podem influenciá-la, como analgesia e adesão (Pekyavaş et al., 2014; Tsai et al., 2009); e em um estudo (Torres-Lacomba, 2020) houve redução significativa de sintomas associados, mas sem diferenças entre os grupos.

\section{Discussão}

O linfedema relacionado ao câncer de mama (LRCM) é uma complicação crônica e progressiva ocasionada por alterações estruturais do sistema linfático que, por sua vez, acarretam um desequilíbrio da função linfática caracterizado pela concentração de linfa nos espaços intersticiais. Ainda não há métodos validados para identificar o risco aumentado para o LRCM e seu tratamento demanda custos elevados. A ausência da possibilidade de cura é uma consequência do pouco entendimento sobre sua fisiopatologia (Invernizzi et al. 2020; Jensen et al. 2015; Ly et al. 2017).

O fluxo linfático é impulsionado pela contração e relaxamento dos músculos (bomba muscular). No entanto, pessoas em tratamento oncológico tendem a reduzir a realização de atividade física devido à fadiga oncológica. A diminuição da mobilidade restringe a ação da bomba muscular, que pode favorecer a evolução do LRCM e piora dos sintomas. A seguir, o LRCM restringe ainda mais os movimentos; manifestando-se um ciclo que apequena a qualidade de vida das pessoas que apresentam o LRCM com isolamento social e desordens emocionais (Malicka et al., 2014; Tantawy, 2019).

O Linfedema pode se apresentar desde o início do tratamento do câncer de mama ou até mesmo após décadas de sua conclusão. Devido seu perfil progressivo, o tratamento correto do LRCM deve ser iniciado precocemente. A Terapia Física Complexa (TFC) é a conduta ouro para o LRCM: o enfaixamento compressivo multicamadas gera um aumento da pressão intersticial e impulsiona a bomba muscular. Entretanto, este tratamento é paliativo e motiva pesquisas que abordem terapias com perspectivas de aumentar sua eficácia (Do et al., 2017; Ergin et al., 2019; Malicka et al., 2014).

O Linfotaping ${ }^{\circledR}$ aparece nesse cenário como uma oportunidade terapêutica alternativa. Ele favorece a função linfática continuamente através da convolução: a fita adesiva aumenta o espaço entre a pele e o músculo, promovendo aumento do fluxo sanguíneo e linfático; absorção do líquido intersticial e da linfa pela diferença de pressão que se forma; e guia a linfa na direção 
desejada. Além disso, inibe as terminações nervosas livres e estimula a analgesia (Kasawara et al., 2018; Thomaz et al. 2018; Wallis et al. 2003; Kase, 2003, 2013).

Uma benesse do Linfotaping ${ }^{\circledR}$ é que a fita adesiva é à prova d'água e pode permanecer por cerca de três dias na pele, propiciando uma ação contínua e sem a necessidade de ser refeito após o banho ou outras atividades diárias. Ademais, tem se mostrado mais confortável e melhor aceito pelos pacientes do que o enfaixamento compressivo multicamadas e as vestes compressivas da TFC, pois permite uma boa mobilidade na realização das atividades diárias e pode ser usado em regiões de climas quente e úmido (Cai et al., 2021; Malicka et al., 2014; Pajero Otero et al., 2019; Tantawy, 2019; Tantawy et al., 2019; Tsai et al., 2009).

A desvantagem é o risco de inflamação e infecção que a pele do membro com LRCM apresenta, o qual pode ser potencializado por alterações dermatológicas provenientes da aplicação do Linfotaping ${ }^{\circledR}$. As alterações dérmicas relatadas parecem estar relacionadas ao uso prolongado; manuseio incorreto das fitas no momento de retirá-las; ou ainda ao uso de fitas semelhantes, mas com qualidade e propriedades diferentes das do Método Kinesio Taping ${ }^{\circledR}$. Mas as respostas dermatológicas são incomuns, e o Linfotaping ${ }^{\circledR}$ é considerado um método seguro e bem aceito pela maioria dos pacientes (Kase et al., 2013; Ergin et al., 2019; de Carvalho Martins et al., 2015; Pajero Otero et al., 2019).

No entanto, evidências científicas sólidas sobre a eficácia do Linfotaping ${ }^{\circledR}$ como tratamento alternativo para o LRCM ainda são limitadas e com resultados conflitantes, o que torna a realização desta revisão pertinente.

Alguns dos estudos analisados apresentaram benefícios do Linfotaping ${ }^{\circledR}$ na redução do volume do Linfedema Relacionado ao Câncer de Mama, e sugere-se que o Linfotaping ${ }^{\circledR}$ traz longevidade ao efeito do tratamento do LRCM (Cai et al., 2021; Do et al., 2017; Malicka et al., 2014; Pajero Otero et al., 2019; Pekyavaş et al., 2014; Pop et al., 2014; Tantawy, 2019; Tantawy et al., 2019; Taradaj et al., 2014). Melgaard (2016) mencionou que a redução da circunferência foi pequena nos grupos, mas sugere um melhor resultado para o grupo que tratou com Kinesio Tape ${ }^{\circledR}$. No entanto, certos autores concluíram que o Linfotaping ${ }^{\circledR}$ não foi mais eficaz na redução do volume do LRCM do que as outras terapias propostas (Ergin et al., 2019; de Carvalho Martins et al., 2015; Smykla et al., 2013; Taradaj et al., 2015; Tsai et al., 2009).

Determinadas pesquisas demonstraram resultados proveitosos relacionados à função do braço acometido pelo LRCM, que podem estar conectados à redução do volume do linfedema e da intensidade dos sintomas associados - espacialmente da dor. Uma das possibilidades para essa analgesia é a "Teoria do Controle de Portas": a fita usada para o Linfotaping ${ }^{\circledR}$ estimula a via neuromuscular por meio de um aumento no feedback aferente, estimulando as fibras nervosas aferentes de grande diâmetro e diminuindo a passagem do estímulo doloroso pelas fibras nervosas de pequeno diâmetro (Cai et al., 2021; Do et al., 2017; de Carvalho Martins et al., 2015; Pajero Otero et al., 2019; Pop et al., 2014; Tantawy, 2019; Tantawy \& Kamel, 2015; Tantawy et al., 2019). Todavia, Taradj (2015) não encontrou evidências que o Linfotaping ${ }^{\circledR}$ seja mais eficaz na melhora da função do que os outros métodos testados, e Smykla (2013) não encontrou evidências de que o Linfotaping ${ }^{\circledR}$ melhora a amplitude de movimento em longo prazo nessa população.

O LRCM impacta tanto fisicamente quanto emocionalmente na qualidade de vida dos sujeitos afetados por ele, podendo gerar angústia, ansiedade e depressão. Trabalhos incluídos nesta revisão mostraram que o Linfotaping ${ }^{\circledR}$ melhora significativamente a qualidade de vida desses sujeitos - pela redução do volume do linfedema e dos sintomas associados, e melhora da função do braço e das características clínicas do tecido (Cai et al., 2021; Do et al., 2017; Melgaard et al., 2016; Pekyavaş et al., 2014; Pop et al., 2014; Tantawy et al., 2019). Torres-Lacomba (2020) relatou que o Linfotaping ${ }^{\circledR}$ foi mais confortável que as outras técnicas analisadas; e que houve redução significativa da sensação de peso e aperto, mas sem diferenças entre os grupos. Tsai (2009) sugere que o Linfotaping ${ }^{\circledR}$ é mais aceito do que a Terapia Física Complexa, promovendo maior conforto, conveniência e adesão dos pacientes. Tantawy e Kamel (2015) observaram uma redução significativa do quadro álgico 
dos ombros dos sujeitos e melhora da função do braço com o uso do Kinesio Tape ${ }^{\circledR}$, o que se acredita influenciar positivamente para recobrar a qualidade de vida.

Alguns fatores relacionados às diferenças metodológicas empregadas nos estudos podem ter contribuído para a diversidade de resultados encontrados. Certos aspectos associados à população - amostras de tamanhos reduzidos e variados (de um a cento e quarenta e seis sujeitos); estágios da evolução do linfedema e fases de tratamento desconhecidos, diversos, ou com evolução indefinida ao longo da pesquisa; incerteza sobre as condições prévias da pele dos sujeitos, como a existência de cicatrizes extensas que alterem a função linfática; disparidade na adesão dos sujeitos ao tratamento, por exemplo, a realização de condutas domiciliares e tempo de uso das terapias propostas.

Condições relativas ao tratamento - quantidade e duração da aplicação das fitas adesivas; tempo entre as substituições delas; locais e métodos de ministração do Linfotaping ${ }^{\circledR}$; valores das pressões aplicadas e complexidade para esse controle; espaço de tempo do tratamento; heterogeneidade das terapias adotadas, tanto nos grupos controle quanto intervenção (tipo de compressão, realização de exercícios e condutas domiciliares).

A região mais tratada foi o antebraço/braço (Cai et al., 2021; Do et al., 2017; Malicka et al., 2014; de Carvalho Martins et al., 2015; Pajero Otero et al., 2019; Pekyavaş et al., 2014; Pop et al., 2014; Smykla et al., 2013; Tantawy, 2019; Tantawy et al., 2019; Taradaj et al., 2014; Taradaj, et al., 2015; Torres-Lacomba et al., 2020); seguida da anastomose axilo-axilar (Ergin et al., 2019; Malicka et al., 2014; de Carvalho Martins et al., 2015; Pajero Otero et al., 2019; Pekyavaş et al., 2014; Pop et al., 2014; Smykla et al., 2013; Tantawy, 2019; Tantawy et al., 2019; Taradaj et al., 2014; Taradaj, et al., 2015); punho/mão (Cai et al., 2021; Do et al., 2017; de Carvalho Martins et al., 2015; Pajero Otero et al., 2019; Pekyavaş et al., 2014; Pop et al., 2014; Smykla et al., 2013; Tantawy, 2019; Tantawy et al., 2019; Taradaj, et al., 2015; Torres-Lacomba et al., 2020); anastomose inguinalaxilar (Smykla et al., 2013; Taradaj et al., 2014; Taradaj, et al., 2015); e cintura escapular (Tantawy \& Kamel, 2015). A direção de aplicação mais comum foi de proximal para distal (Cai et al., 2021; Ergin et al., 2019; Malicka et al., 2014; de Carvalho Martins et al., 2015; Pajero Otero et al., 2019; Pekyavaş et al., 2014; Pop et al., 2014; Smykla et al., 2013; Tantawy, 2019; Tantawy et al., 2019; Taradaj et al., 2014; Taradaj, et al., 2015; Torres-Lacomba et al., 2020); e a direção distal para proximal foi usada no grupo intervenção no estudo de Pop (2014). Em duas pesquisas - Ergin (2019) e Tantawy \& Kamel (2015) - não foi possível afirmar a direção de aplicação das fitas.

O alongamento das caudas variou de ausência de tensão à 15\% (Ergin et al., 2019; Malicka et al., 2014; Pajero Otero V et al., 2019; Pop TB et al., 2014; Smykla et al., 2013; Taradaj J et al., 2014; Taradaj, et al., 2015) e sem tensão nas regiões do processo espinhoso da escápula, redondo menor e deltóide, conforme Tantawy e Kamel (2015). A tensão das caudas foi de 15\% à 40\% (Ergin G et al., 2019; Tantawy \& Kamel, 2015; Taradaj J et al., 2014; Tsai et al., 2009) e em um estudo essa tensão foi usada apenas para o supraespinhal, em Tantawy e Kamel (2015); tensão maior que 50\% para correção mecânica do deltóide posterior (Tantawy \& Kamel, 2015). Em três estudos não foi possível afirmar a tensão do alongamento das caudas (Cai H et al., 2021; de Carvalho Martins et al., 2015; Pekyavaş et al., 2014). Quanto à aplicação das âncoras, houve uma predileção pelo método sem tensão. Em duas pesquisas não foi possível identificar os métodos, locais, direções e tensões de aplicação do Linfotaping $^{\circledR}$ (Melgaard et al., 2016; Tsai et al., 2009).

A quantidade de aplicações de Linfotaping ${ }^{\circledR}$ foi menor que dez em onze estudos (Ergin et al., 2019; Malicka et al., 2014; de Carvalho Martins et al., 2015; Melgaard et al., 2016; Pajero Otero et al., 2019; Pop et al., 2014; Tantawy, 2019; Tantawy \& Kamel, 2015; Tantawy et al., 2019; Taradaj et al.; 2014, 2015), e igual ou maior a dez em seis deles (Cai et al., 2021; Do et al., 2017; Pekyavaş et al., 2014; Smykla et al., 2013; Torres-Lacomba et al., 2020; Tsai et al., 2009). O tempo de tratamento dos sujeitos variou de quatro dias a quatro semanas.

Circunstâncias concernentes aos desenhos e execução dos estudos também podem ter influenciado nas divergências dos resultados - diferentes recursos de mensuração das variáveis; múltiplos pesquisadores aplicando as terapias em um mesmo 
sujeito; o mesmo investigador realizando tratamento e avaliação; diferentes desenhos de estudo; dificuldade em cegar os sujeitos, pois as fitas são visíveis e com propriedades físicas características; e a complexidade para realizar um grupo controle real, por questões éticas de cada País.

Perimetria (Cai et al., 2021; Ergin et al., 2019; de Carvalho Martins et al., 2015; Melgaard et al., 2016; Tantawy, 2019), volumetria indireta por perimetria (Do et al., 2017; Malicka et al., 2014; Pajero Otero et al., 2019; Pekyavaş et al., 2014; Tantawy et al., 2019; Torres-Lacomba et al., 2020), perimetria e deslocamento de água (Tsai HJ et al., 2009), Perímetro Optoeletrônico 400T (Smykla et al., 2013; Taradaj et al., 2014; Taradaj, et al., 2015) e um instrumento de autoria própria (Pop et al., 2014) foram usados como recursos para avaliar o volume.

Goniometria foi utilizada em Pajero Otero (2019), Pop (2014) e Taradaj (2015), Questionário Quick DASH Outcome Measure em Cai (2021), Do (2017) e de Carvalho Martins (2015) e Índice de Dor e Incapacidade no Ombro / SPADI foram utilizados como instrumento para mensurar a função do braço acometido pelo LRCM em Tantawy e Kamel (2015) e Tantawy (2019).

Foram empregados para medir a qualidade de vida dos sujeitos afetados pelo LRCM o questionário EORTC QLQ-C30 em Do (2017) e Tantawy (2019), EORTC OLQ-C30 associado ao EORTC QLQ-BR23 em Tsai (2009), Escala de Qualidade de Vida da Organização Mundial de Saúde / WHOQOL-BREF em Cai (2021), questionário Short-Form 36 em Pekyavaş (2014), e entrevista pós-tratamento.

Ademais, os estudos não abordam as condições sazonais em que as pesquisas foram realizadas, dificultando a padronização do tratamento para as diversidades climáticas. E há uma carência de acompanhamento dos sujeitos para avaliação dos efeitos em longo prazo.

Atualmente, o uso do Linfotaping ${ }^{\circledR}$ no tratamento do Linfedema Relacionado ao Câncer de Mama tem apresentado indicadores positivos na redução do volume, melhora da função do membro acometido, e principalmente na recuperação da qualidade de vida dos sujeitos abalados pelo LRCM. Mas sua aplicação ainda tem sido limitada à complementar o método padrão ouro - Terapia Física Complexa (TFC). Caso uma eficácia semelhante à da TFC seja demonstrada, o emprego do Linfotaping ${ }^{\circledR}$ poderá otimizar os recursos por ser mais barato e demandar menor frequência de aplicação que a TFC; e possibilitará uma nova modalidade terapêutica para pacientes que têm contraindicações ou baixa tolerância ao tratamento padrão-ouro vigente.

\section{Conclusão}

A possibilidade de prevenção e tratamento curativo para o Linfedema Relacionado ao Câncer de Mama está sujeita ao conhecimento dos mecanismos celulares e moleculares relacionados a esta morbidade; assim como a atuação das alternativas terapêuticas na sua fisiopatologia - entre elas o Linfotaping ${ }^{\circledR}$. Através desta revisão de literatura foi observado que o Linfotaping ${ }^{\circledR}$ pode promover efeitos benéficos para o volume e funcionalidade dos braços acometidos pelo LRCM, assim como para a qualidade de vida de mulheres afetadas por este tipo de linfedema.

No entanto, ensaios clínicos randomizados adicionais são necessários para que se tenham evidências sólidas sobre o uso do Linfotaping ${ }^{\circledR}$ como terapia alternativa efetiva para o LRCM e especificar em quais situações o Linfotaping ${ }^{\circledR}$ se mostra mais eficaz. A população precisa ser mais bem definida, com amostragem maior e descrição dos estágios do linfedema e fases do tratamento abordados; metodologias mais descritivas, com especificações do modo de aplicar as fitas, frequência de aplicação, tempo de tratamento, a tolerância dos pacientes e a qualidade do material utilizado. Além disso, sugere-se analisar a influência do Linfotaping ${ }^{\circledR}$ em outros parâmetros clínicos e laboratoriais que possam estar relacionados ao LRCM.

Portanto, a continuação de estudos que reforcem a comprovação da eficácia do Linfotaping ${ }^{\circledR}$ no tratamento do linfedema relacionado ao câncer de mama, com repercussão expressiva na prática clínica, promoverá a qualidade de vida destes pacientes e reduzirá gastos com assistência de saúde. 


\section{Referências}

Azhar, S. H., Lim, H. Y., Tan, B. K., \& Angeli, V. (2020). The unresolved pathophysiology of lymphedema. Frontiers in physiology, $11,137$.

Cai, H., Wang, W. \& Zhou, X. (2021). Kinesiology taping combined with manual lymph drainage reduces postoperative lymphedema related to breast cancer. Chinese Journal of Tissue Engineering Research, 25(14), 2247.

de Carvalho Martins, J., Aguiar, S. S., Fabro, E. A. N., Costa, R M., Lemos, T. V., de Sá, V. G. G., \& Bergmann, A. (2016). Safety and tolerability of Kinesio® Taping in patients with arm lymphedema: medical device clinical study. Supportive care in cancer, 24(3), 1119-1124.

Do, J., Jeon, J. \& Kim, W. (2017). The effects of bandaging with an additional pad and taping on secondary arm lymphedema in a patient after mastectomy. Journal of physical therapy science, 29(7), 1272-1275.

Ergin, G., Şahinoğlu, E., Karadibak, D. \& Yavuzşen, T. (2019). Effectiveness of Kinesio Taping on anastomotic regions in patients with breast cancer-related lymphedema: a randomized controlled pilot study. Lymphatic research and biology, 17(6), 655-660.

Ezzo, J., Manheimer, E., McNeely, M. L., Howell, D. M., Weiss, R., Johansson, K. I., \& Karadibak, D. (2015). Manual lymphatic drainage for lymphedema following breast cancer treatment. Cochrane Database of Systematic Reviews, (5).

Finnane, A., Janda, M. \& Hayes, S. C. (2015). Review of the evidence of lymphedema treatment effect. American journal of physical medicine \& rehabilitation, 94(6), 483-498.

Gatt, M., Willis, S. \& Leuschner, S. (2017). A meta-analysis of the effectiveness and safety of kinesiology taping in the management of cancer-related lymphoedema. European journal of cancer care, 26(5), e12510.

Invernizzi, M., Lopez, G., Michelotti, A., Venetis, K., Sajjadi, E., Mattos-Arruda, D., \& Fusco, N. (2020). Integrating biological advances into the clinical Management of Breast Cancer Related Lymphedema. Frontiers in oncology, 10, 422.

Jensen, M. R., Simonsen, L., Karlsmark, T., Lanng, C. \& Bülow, J. (2015). Higher vascular endothelial growth factor-C concentration in plasma is associated with increased forearm capillary filtration capacity in breast cancer-related lymphedema. Physiological reports, 3(6), e12403.

Kasawara, K. T., Mapa, J. M. R., Ferreira, V., Added, M. A. N., Shiwa, S. R., Carvas Jr, N. \& Batista, P. A. (2018). Effects of Kinesio Taping on breast cancerrelated lymphedema: A meta-analysis in clinical trials. Physiotherapy theory and practice, 34(5), 337-345.

Kase, K. (2003). Illustrated Kinesio Taping. (4a ed.) Tokyo, Japan: Ken Ikai Co Ltd.

Kase, K., Lemos, T. V., \& Dias, E. M. (2013). Kinesio Taping: introdução ao método e aplicações musculares. São Paulo: Andreoli, 11-12.

Leung, N., Furniss, D. \& Giele, H. (2015). Modern surgical management of breast cancer therapy related upper limb and breast lymphoedema. Maturitas, 80(4), 384-390.

Li, C. Y., Kataru, R. P. \& Mehrara, B. J. (2020). Histopathologic features of lymphedema: A molecular review. International journal of molecular sciences, $21(7), 2546$.

Li, L., Yuan, L., Chen, X., Wang, Q., Tian, J., Yang, K. \& Zhou, E. (2016). Current treatments for breast cancer-related lymphoedema: a systematic review. Asian Pacific journal of cancer prevention: APJCP, 17(11), 4875.

Ly, C. L., Kataru, R. P. \& Mehrara, B. J. (2017). Inflammatory manifestations of lymphedema. International journal of molecular sciences, $18(1), 171$.

Malicka, I., Rosseger, A., Hanuszkiewicz, J. \& Woźniewski, M. (2014). Kinesiology Taping reduces lymphedema of the upper extremity in women after breast cancer treatment: a pilot study. Przeglad menopauzalny= Menopause review, 13(4), 221.

Marx, A. \& Figueira, P. (2017). Fisioterapia no Câncer de Mama.

Melgaard, D. (2016). What is the effect of treating secondary lymphedema after breast cancer with complete decongestive physiotherapy when the bandage is replaced with Kinesio Textape?-A pilot study. Physiotherapy theory and practice, 32(6), 446-451.

Pajero Otero, V., Garcia Delgado, E., Martin Cortijo, C., Romay Barrero, H. M., de Carlos Iriarte, E. \& Avendaño-Coy, J. (2019). Kinesio taping versus compression garments for treating breast cancer-related lymphedema: a randomized, cross-over, controlled trial. Clinical rehabilitation, 33(12), $1887-1897$.

Pekyavaş, N. Ö., Tunay, V. B., Akbayrak, T., Kaya, S. \& Karataş, M. (2014). Complex decongestive therapy and taping for patients with postmastectomy lymphedema: a randomized controlled study. European Journal of Oncology Nursing, 18(6), 585-590.

Poage, E. G., Rodrick, J. R., Wanchai, A., Stewart, B. R., Cormier, J. N. \& Armer, JM. (2015). Exploring the usefulness of botanicals as an adjunctive treatment for lymphedema: a systematic search and review. $P M \& R, 7(3), 296-310$.

Pop, T. B., Karczmarek-Borowska, B., Tymczak, M., Hałas, I. \& Banaś, J. (2014). The influence of Kinesiology Taping on the reduction of lymphoedema among women after mastectomy-preliminary study. Contemporary oncology, 18(2), 124.

Rezende, L., Campanholi, L. L. \& Tessaro, A. (2018). Manual de Condutas e Práticas Fisioterapêuticas no Câncer de Mama da ABFO. Thieme Revinter Publicações LTDA.

Singh, B., Disipio, T., Peake, J. \& Hayes, S. C. (2016). Systematic review and meta-analysis of the effects of exercise for those with cancer-related lymphedema. Archives of physical medicine and rehabilitation, 97(2), 302-315. 
Research, Society and Development, v. 11, n. 2, e28411224973, 2022

(CC BY 4.0) | ISSN 2525-3409 | DOI: http://dx.doi.org/10.33448/rsd-v11i2.24973

Smile, T. D., Tendulkar, R., Schwarz, G., Arthur, D., Grobmyer, S., Valente, S., \& Shah, C. (2018). A review of treatment for breast cancer-related lymphedema. American journal of clinical oncology, 41(2), 178-190.

Smoot, B., Chiavola-Larson, L., Lee, J., Manibusan, H. \& Allen, D. D. (2015). Effect of low-level laser therapy on pain and swelling in women with breast cancer-related lymphedema: a systematic review and meta-analysis. Journal of Cancer Survivorship, 9(2), 287-304.

Smykla, A., Walewicz, K., Trybulski, R., Halski, T., Kucharzewski, M., Kucio, C. \& Taradaj, J. (2013). Effect of kinesiology taping on breast cancer-related lymphedema: a randomized single-blind controlled pilot study. BioMed research international, 2013.

Tantawy, S. A. (2019). Secondary upper limb lymphedema following mastectomy responses to kinesio taping application: A pilot study. Bioscience Research, $16(2), 1174-1180$

Tantawy, S. A. \& Kamel, D. M. (2016). The effect of kinesio taping with exercise compared with exercise alone on pain, range of motion, and disability of the shoulder in postmastectomy females: a randomized control trial. Journal of physical therapy science, 28(12), 3300-3305.

Tantawy, S. A., Abdelbasset, W. K., Nambi, G. \& Kamel, D. M. (2019). Comparative study between the effects of kinesio taping and pressure garment on secondary upper extremity lymphedema and quality of life following mastectomy: a randomized controlled trial. Integrative cancer therapies, 18, 1534735419847276.

Taradaj, J., Halski, T., Zduńczyk, M., Rajfur, J., Pasternok, M., Chmielewska, D., Piecha, M., Kwasna, K. \& Skrzypulec-Plinta, V. (2014). Evaluation of the effectiveness of kinesio taping application in a patient with secondary lymphedema in breast cancer: a case report. Przeglad menopauzalny= Menopause review, $13(1), 73$

Taradaj, J., Halski, T., Rosinczuk, J., Dymarek, R., Laurowski, A. \& Smykla, A. (2015). The influence of Kinesiology Taping on the volume of lymphoedema and manual dexterity of the upper limb in women after breast cancer treatment. European Journal of Cancer Care, 25(4), 647-660.

Thomaz, J. P., Dias, T. D. S. M., \& Rezende, L. F. D. (2018). Effect of taping as treatment to reduce breast cancer lymphedema: literature review. Jornal vascular brasileiro, 17, 136-140

Torres-Lacomba, M., Navarro-Brazález, B., Prieto-Gómez, V., Ferrandez, J. C., Bouchet, J. Y. \& Romay-Barrero, H. (2020). Effectiveness of four types of bandages and kinesio-tape for treating breast-cancer-related lymphoedema: a randomized, single-blind, clinical trial. Clinical Rehabilitation, 34(9), 1230-1241.

Tsai, H. J., Hung, H. C., Yang, J. L., Huang, C. S. \& Tsauo, J. Y. (2009). Could Kinesio tape replace the bandage in decongestive lymphatic therapy for breastcancer-related lymphedema? A pilot study. Supportive care in cancer, 17(11), 1353-1360.

Wallis, J., Kase, T. \& Kase, K. (2003). Clinical therapeutic applications of the kinesio taping method. Albuquerque .

Wetzig, N., Gill, P. G., Espinoza, D., Mister, R., Stockler, M. R., Gebski, V. J., Ung, O. A., Campbell, I. \& Simes, J. (2017). Sentinel-lymph-node-based management or routine axillary clearance? Five-year outcomes of the RACS Sentinel Node Biopsy Versus Axillary Clearance (SNAC) 1 Trial: assessment and incidence of true lymphedema. Annals of surgical oncology, 24(4), 1064-1070.

Yuan, Y., Arcucci, V., Levy, S. M., \& Achen, M. G. (2019). Modulation of immunity by lymphatic dysfunction in lymphedema. Frontiers in immunology, 10, 76. 